\title{
A Nonlinear Multiparameters Temperature Error Modeling and Compensation of POS Applied in Airborne Remote Sensing System
}

\author{
Jianli Li, ${ }^{1,2}$ Wenjian Wang, ${ }^{1,2}$ Feng Jiao, ${ }^{1,2}$ Jiancheng Fang, ${ }^{1,2}$ and Tao Yu ${ }^{3}$ \\ ${ }^{1}$ School of Instrument Science and Opto-Electronic Engineering, Beijing University of Aeronautics and Astronautics, \\ Beijing 100191, China \\ ${ }^{2}$ Science \& Technology on Inertial Laboratory, Key Laboratory of Fundamental Science for National Defense-Novel \\ Inertial Instrument \& Navigation System Technology, Beijing University of Aeronautics and Astronautics, Beijing 100191, China \\ ${ }^{3}$ Xian Institute of Optics and Precision Mechanics (XIOPM), CAS, Xian 710119, China
}

Correspondence should be addressed to Wenjian Wang; desa001@sina.com

Received 10 March 2014; Accepted 13 May 2014; Published 9 June 2014

Academic Editor: Guiming Luo

Copyright (C) 2014 Jianli Li et al. This is an open access article distributed under the Creative Commons Attribution License, which permits unrestricted use, distribution, and reproduction in any medium, provided the original work is properly cited.

The position and orientation system (POS) is a key equipment for airborne remote sensing systems, which provides high-precision position, velocity, and attitude information for various imaging payloads. Temperature error is the main source that affects the precision of POS. Traditional temperature error model is single temperature parameter linear function, which is not sufficient for the higher accuracy requirement of POS. The traditional compensation method based on neural network faces great problem in the repeatability error under different temperature conditions. In order to improve the precision and generalization ability of the temperature error compensation for POS, a nonlinear multiparameters temperature error modeling and compensation method based on Bayesian regularization neural network was proposed. The temperature error of POS was analyzed and a nonlinear multiparameters model was established. Bayesian regularization method was used as the evaluation criterion, which further optimized the coefficients of the temperature error. The experimental results show that the proposed method can improve temperature environmental adaptability and precision. The developed POS had been successfully applied in airborne TSMFTIS remote sensing system for the first time, which improved the accuracy of the reconstructed spectrum by $47.99 \%$.

\section{Introduction}

The position and orientation system (POS) is a special strapdown inertial navigation system (SINS)/global positioning system (GPS) integrated measurement equipment [1]. Compared with the traditional SINS/GPS integrated system, it is small, lightweight, and is also available to provide highprecision position, velocity, and attitude information for airborne remote sensing applications including spectrometer (such as Temporally and Spatially Modulated Fourier Transform Imaging Spectrometer (TSMFTIS)), Synthetic Aperture Radar (SAR), Light Detection and Ranging (LiDAR), optical camera, and various other land and marine applications. It has been widely used as a key equipment to further improve imaging quality and efficiency of airborne remote sensing systems [2-4]

However, the precision of POS decreases rapidly with the environmental temperature change [5]. Therefore, it is vital to model and compensate the temperature error of POS. Traditional temperature error modeling and compensation for POS include two kinds of methods: one is polynomial fitting based on least square or multiple regression algorithm to establish the relationship between the temperature input and the POS output [6]. These methods can diminish computational complexity and can be suitable for realtime signal processing of simple model, which are used to compensate temperature error of a traditional SINS/GPS integrated system. However, their precision is limited because 


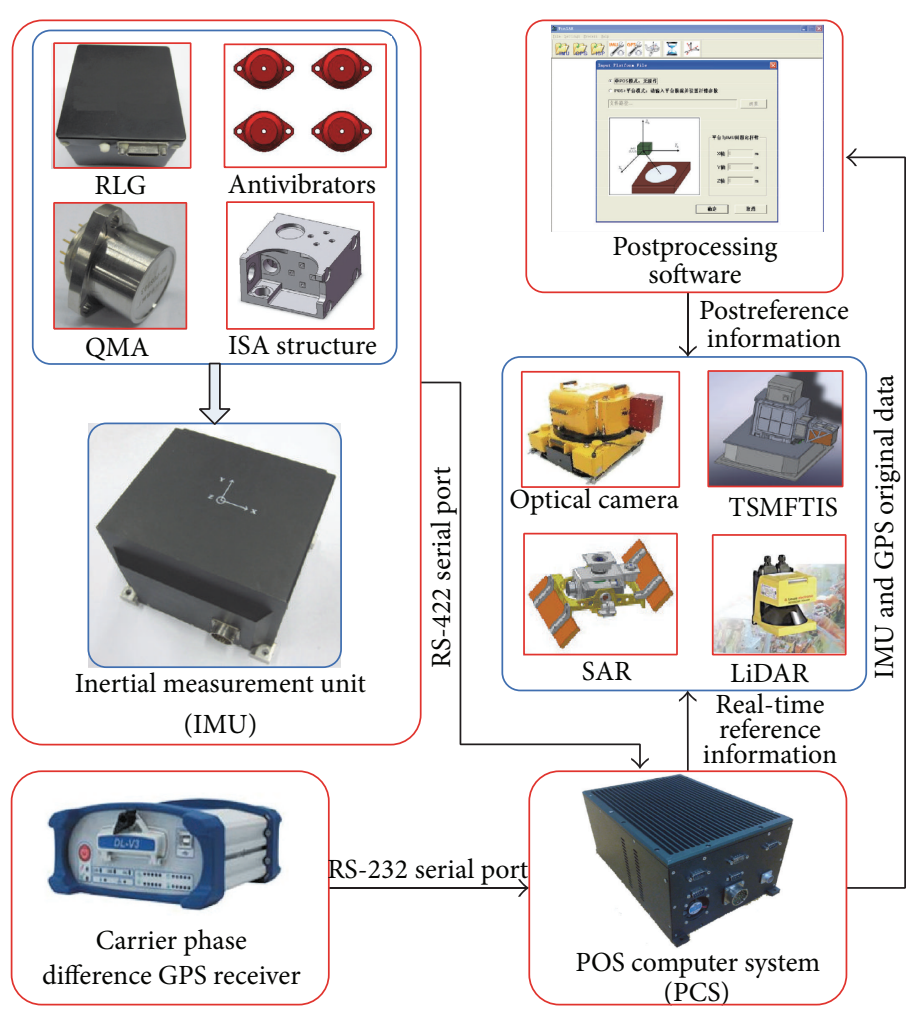

FIGURE 1: The components and operation principle of POS.

the model only considers the single temperature without sufficient parameters. The other is based on machine learning technique, such as support vector machine (SVM) [7] and radial basis function (RBF) neural network [8]. In general, $\mathrm{RBF}$ neural network is a massively parallel-distributed processor that can be used in complex model with multiparameters and nonlinear problems [9] and can improve the precision in postprocessing of POS. However, traditional RBF neural network method is barely applied to new samples because of its poor generalization ability, which leads to the low repeatability of the compensated results under different temperature condition [10].

Therefore, how to establish a sufficient model and improve the generalization ability has become a key technology for modeling and compensating temperature error of POS. To solve this problem, a nonlinear multiparameters temperature error modeling and compensation method based on Bayesian regularization neural network is proposed in this paper. All the influence factors including temperature, rate of temperature change, and temperature gradient are considered to establish a sufficient multiparameters and nonlinear temperature error model. Bayesian regularization is regarded as evaluation criterion to optimize the coefficients of the temperature error for POS, which further improves the generalization ability. Temperature experiment is implemented to validate the proposed method. The first airborne application experiment of TSMFTIS proves that TSMFTIS has an evident improvement on imaging quality and precision of reconstructed spectrum by using POS data.
This paper is organized as follows. Section 2 introduces the components and operation principle of POS. In Section 3, the temperature error modeling and compensation method of POS based on Bayesian regularization neural network is proposed. In Section 4, the temperature experiment result is presented and validates effectiveness of the proposed method. The airborne experiment of TSMFTIS with POS is introduced in Section 5. Finally, the paper is concluded in Section 6.

\section{The Components and Operation Principle of POS}

POS is comprised of four main components which are inertial measurement unit (IMU), carrier phase differential GPS receiver, POS computer system (PCS), and postprocessing software as shown in Figure 1.

IMU is mounted directly onto imaging payloads and measures the motion rotation rate and the linear accelerometer information, which determines the precision of POS directly [11]. IMU mainly consists of three ring laser gyroscopes (RLG) and three quartz mechanical accelerometers (QMA) assembled in inertial sensing assembly (ISA) structure in orthogonal triads [12]. The ISA with inertial sensors is installed on exterior supporting frame by eight antivibrators.

A carrier phase differential GPS receiver can provide time, position, and velocity information. PCS is a multifunctional computer system and mainly completes the IMU data sampling and storage, data synchronization, real-time integrated navigation computing, and communication with 


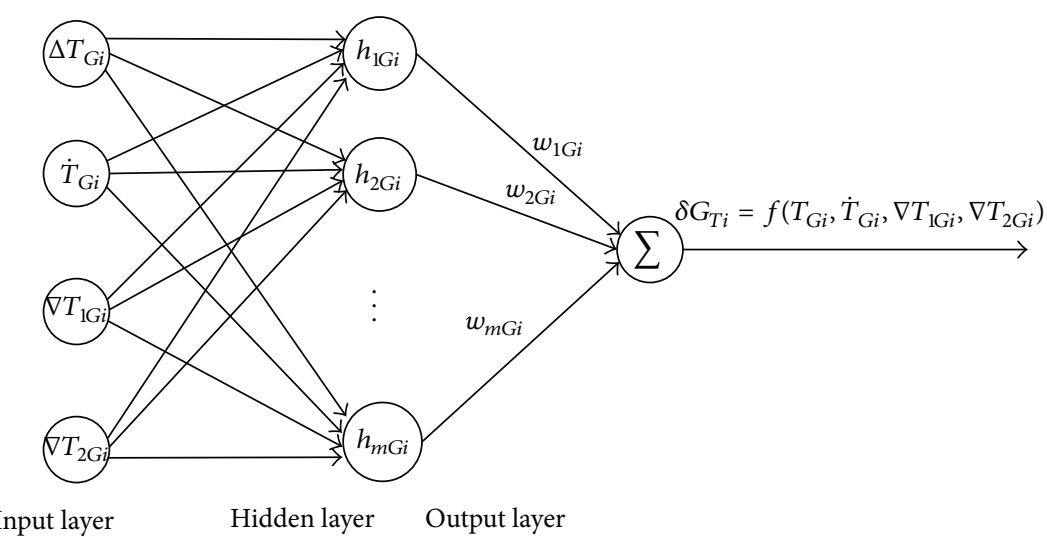

FIGURE 2: Temperature error modeling for gyroscope assembly based on RBF neural network.

other systems. The integrated system of POS provides excellent short-term dynamics greatly and has none of the longterm drift problem associated with inertial measurement system. The result can be obtained in real time by PCS or in postprocessing by postprocessing software. The postprocessing algorithm in software is available to calculate complex model of massive computation without time limit, which can further improve the precision of POS. As a result, POS can successively provide high-precision position, velocity, and attitude information to improve imaging quality and efficiency for airborne remote sensing system [13].

\section{Temperature Error Modeling and Compensation of POS}

The output errors of gyroscope assembly in angular rate channel for POS mainly include bias and scale factor error. For RLG, scale factor error varies little with the change of temperature, while bias presents the opposite property [14]. Hence, the temperature error of gyroscope assembly mainly refers to the temperature error of gyroscope bias influenced by the temperature, rate of temperature change, and temperature gradient. The output errors of accelerometer assembly in linear acceleration channel for POS include bias and scale factor error. Temperature changes have a great influence on bias of accelerometers. All these properties mentioned above make it necessary to compensate bias temperature errors of gyroscope and accelerometer assemblies to improve the efficiency and precision of POS.

3.1. Nonlinear Multiparameters Temperature Error Modeling of Gyroscope Assembly. Three RLGs are assembled orthogonally in ISA structure of IMU. The RLG in the $i$ th $(i=x, y, z)$ axis embeds three thermometers, which measure the temperature of anode $\left(T_{a G i}\right)$, cathode $\left(T_{c G i}\right)$, and shell $\left(T_{s G i}\right)$, respectively. The temperature, which may result in thermal deformation of different materials in RLG, will induce the change of light path of RLG [8]. The temperature gradient, exaggerated by the rapid change of external temperature, also affects the output of RLG since it leads to extrusion and deflection of components in RLG. All these factors should be considered in the temperature error model of gyroscope assembly for POS.

In order to establish the sufficient temperature error model of gyroscope assembly, the multiple parameters including temperature, temperature change, and temperature gradient should be considered. Therefore, the input $\mathbf{X}_{G i}$ of gyroscope in the $i$ th axes is given as follows:

$$
\mathbf{X}_{G i}=\left[\begin{array}{c}
T_{G i} \\
\dot{T}_{G i} \\
\nabla T_{1 G i} \\
\nabla T_{2 G i}
\end{array}\right]=\left[\begin{array}{c}
\frac{T_{a G i}+T_{c G i}+T_{s G i}}{3} \\
\frac{\dot{T}_{a G i}+\dot{T}_{c G i}+\dot{T}_{s G i}}{3} \\
T_{a G i}-T_{s G i} \\
T_{c G i}-T_{s G i}
\end{array}\right],
$$

where temperature $T_{G i}$ is the average of the three temperature values for gyroscope in the $i$ th axes, which has been smoothed to estimate the measurement error. The rate of temperature change $\dot{T}_{G i}$ is the derivative of $T_{G i}$. Limited by the measurement condition, temperature gradients $\nabla T_{1 G i}$ and $\nabla T_{2 G i}$ are replaced by the temperature differences $T_{a G i}$ and $T_{c G i}$ from $T_{s G i}$, respectively, because $T_{s G i}$ is the closest to the external environment temperature.

Therefore, the nonlinear multiparameters temperature error model of gyroscope in the $i$ th axes can be expressed qualitatively as the function of the temperature input $\mathbf{X}_{G i}$ :

$$
\delta \bar{G}_{T i}=f\left(\mathbf{X}_{G i}\right)=f\left(\left[T_{G i}, \dot{T}_{G i}, \nabla T_{1 G i}, \nabla T_{2 G i}\right]^{T}\right) .
$$

The RBF neural network is a special feed-forward network and commonly has three layers: an input layer, a single hidden layer, and an output layer [15]. The hidden layer transfers the linear model into a nonlinear one and maps the input space to a new solution space. According to the characteristic of nonlinear and multiparameters in the temperature error model of gyroscope assembly, the RBF neural network here can be given in Figure 2 .

Radial basis function is usually defined as a function of the Euclidean distance from arbitrary input $\mathbf{X}_{G i}$ to 


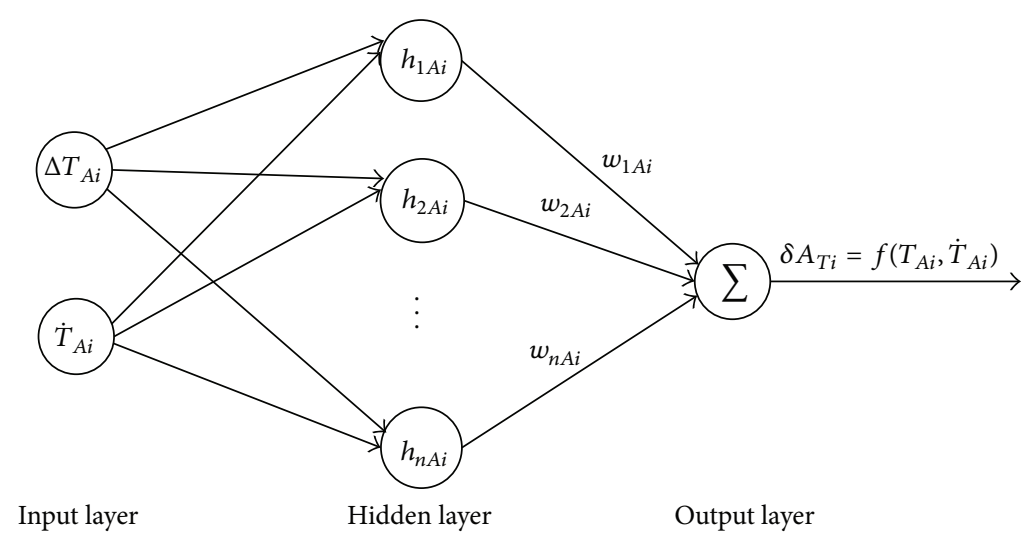

FIGURE 3: Temperature error modeling for accelerometer assembly based on RBF neural network.

the center $\mathbf{C}_{j G i}$; it can be written as $h_{j G i}$. The most common radial basis function is Gauss kernel function:

$$
h_{j G i}=\exp \left\{-\frac{\left\|\mathbf{X}_{G i}-\mathbf{C}_{j G i}\right\|^{2}}{2 \sigma_{j G i}^{2}}\right\},
$$

where $\mathbf{C}_{j G i}$ is the basis function center of the $j$ th node in the hidden layer for gyroscope in the $i$ th axes and $\sigma_{j G i}$ represents the spread of the basis function.

The neural network theory holds that any function can be expressed as a weighted sum of a set of basis functions in solving function approximation problem. So the temperature error model of gyroscope assembly can be given as follows:

$$
\begin{aligned}
\delta \bar{G}_{T i} & =f\left(\mathbf{X}_{G i}\right)=\sum_{j=1}^{M_{G}} w_{j G i} h_{j G i} \\
& =\sum_{j=1}^{M_{G}} w_{j G i} \exp \left\{-\frac{\left\|\mathbf{X}_{G i}-\mathbf{C}_{j G i}\right\|^{2}}{2 \sigma_{j G i}^{2}}\right\},
\end{aligned}
$$

where $w_{j G i}$ is the $j$ th the weight value from the hidden layer to the output layer of gyroscope in the $i$ th axes; $M_{G}$ represents the node number of the hidden layer. $\mathbf{C}_{j G i}, \sigma_{j G i}$, and $w_{j G i}$ can be obtained by training process of RBF neural network during the temperature compensation.

\subsection{Nonlinear Multiparameters Temperature Error Modeling} of Accelerometer Assembly. Unlike RLG, QMA only has one embedded thermometer. The accelerometer assembly temperature error model only can obtain temperature and rate of temperature change without temperature gradient. According to the characteristic of temperature error for accelerometer assembly, the nonlinear multiparameters temperature error model of accelerometer in the $i$ th axes can be expressed qualitatively as the function of the temperature input $\mathbf{X}_{A i}$ :

$$
\delta \bar{A}_{T i}=f\left(\mathbf{X}_{A i}\right)=f\left(\left[T_{A i}, \dot{T}_{A i}\right]^{T}\right) .
$$

The temperature input of accelerometer in the $i$ th axes is $\mathbf{X}_{A i}=\left[\begin{array}{ll}T_{A i} & \dot{T}_{A i}\end{array}\right]^{T}$ with $T_{A i}$ being the output of thermometer embedded in accelerometer in the $i$ th axes; $\dot{T}_{A i}$ is the derivative of $T_{A i}$.

The RBF neural network of accelerometer assembly is given in Figure 3.

The radial basis function is also Gauss kernel function.

The temperature error model of accelerometer assembly can be given as follows:

$$
\begin{aligned}
\delta \bar{A}_{T i} & =f\left(\mathbf{X}_{A i}\right)=\sum_{j=1}^{M_{A}} w_{j A i} h_{j A i} \\
& =\sum_{j=1}^{M_{A}} w_{j A i} \exp \left\{-\frac{\left\|\mathbf{X}_{A i}-\mathbf{C}_{j A i}\right\|^{2}}{2 \sigma_{j A i}^{2}}\right\},
\end{aligned}
$$

where $w_{j A i}$ is the $j$ th the weight value from the hidden layer to the output layer of accelerometer in the $i$ th axes; $M_{A}$ represents the node number of the hidden layer. $\mathbf{C}_{j A i}$ is the basis function center of the $j$ th node in the hidden layer for accelerometer in the $i$ th axes and $\sigma_{j A i}$ represents the spread of the basis function. $\mathbf{C}_{j A i}, \sigma_{j A i}$, and $w_{j A i}$ can be obtained by training process of RBF neural network during the temperature compensation.

\subsection{Temperature Error Compensation Method of RBF Neural} Network Based on Bayesian Regularization. The poor generalization ability is the most troubling problem affecting the application of RBF neural network. In the traditional algorithm of RBF neural network, the mean square error (MSE) is usually used as the evaluation criterion [14]. The MSE of temperature error in gyroscope and accelerometer assemblies can be given as follows:

$$
\begin{aligned}
& E_{d G i}=\frac{1}{N} \sum_{n=1}^{N}\left(f\left(\mathbf{X}_{G i}(n)\right)-\delta \bar{G}_{T i}(n)\right)^{2}, \\
& E_{d A i}=\frac{1}{N} \sum_{n=1}^{N}\left(f\left(\mathbf{X}_{A i}(n)\right)-\delta \bar{A}_{T i}(n)\right)^{2},
\end{aligned}
$$

where $\delta \bar{G}_{T i}(n)$ and $\delta \bar{A}_{T i}(n)$ are the $n$th training target of the sample for gyroscope and accelerometer in the $i$ th 
axes, respectively; $X_{G i}(n)$ and $X_{A i}(n)$ are the corresponding temperature input for gyroscope and accelerometer in the $i$ th axes, respectively; $f\left(\mathbf{X}_{G i}(n)\right)$ and $f\left(\mathbf{X}_{A i}(n)\right)$ are the output of the training sample for gyroscope and accelerometer in the $i$ th axes, respectively; $N$ is the total number of the training sample.

The MSE of the training sample can only reflect the ability to approximate the sample of RBF neural network, not representing the generalization ability. A major issue for traditional RBF neural network methods is the potential for overfitting which leads to a fitting of the noise and loses generalization of the network [16]. To reduce the potential for overfitting, an improved RBF neural network based on Bayesian regularization is proposed to prevent the weight values from growing too large by appending weight values in the evaluation criterion. The smaller the weight values are, the better the generalization capability of the network is. The weight decay regularizer can be given as follows:

$$
\begin{aligned}
& E_{w G i}=\frac{1}{M_{G}} \sum_{k=1}^{M_{G}} w_{j G i}^{2}, \\
& E_{w A i}=\frac{1}{M_{A}} \sum_{k=1}^{M_{A}} w_{j A i}^{2} .
\end{aligned}
$$

So the evaluation criterion of Bayesian regularization can be given as follows:

$$
\begin{aligned}
& F_{G i}\left(w_{G i}\right)=\alpha_{G i} E_{w G i}+\beta_{G i} E_{d G i}, \\
& F_{A i}\left(w_{A i}\right)=\alpha_{A i} E_{w A i}+\beta_{A i} E_{d A i},
\end{aligned}
$$

where $\alpha_{G i}, \beta_{G i}, \alpha_{A i}$, and $\beta_{A i}$ are the evaluation criterion function parameters. Under the situation of $\alpha_{G i} \ll \beta_{G i}$ or $\alpha_{A i} \ll \beta_{A i}$, overfitting may occur due to the overemphasis on reducing training error. While on the other hand, too much concentration on limiting the network weight values when $\alpha_{G i} \gg \beta_{G i}$ or $\alpha_{A i} \gg \beta_{A i}$ may probably lead to large training error. Therefore, how to find optimal values of $\alpha_{G i}$, $\beta_{G i}, \alpha_{A i}$, and $\beta_{A i}$ is a key point of Bayesian regularization. The Bayesian regularization to RBF network training makes use of an iterative procedure in the manner of the expectationmaximization algorithm for estimating the network weights. The iterations consist of finding the most probable value for the weights from their distribution [17]. In the Bayesian framework, the weight values of the network are assumed to be random variables. According to Bayesian's rule [18], the optimization of the regularization parameters $\alpha_{G i}, \beta_{G i}, \alpha_{A i}$, and $\beta_{A i}$ require solving the Hessian matrix of $F_{G i}\left(w_{G i}^{M P}\right)$ and $F_{A i}\left(w_{A i}^{M P}\right)$ at the minimum point $w_{G i}^{M P}$ and $w_{A i}^{M P}$. The optimal weight values $w_{G i}^{M P}$ and $w_{A i}^{M P}$ should maximize the posterior probability; this is equivalent to minimizing the regularized evaluation criterion functions $F_{G i}\left(w_{G i}\right)$ and $F_{A i}\left(w_{A i}\right)$.

Initializing the value of $\alpha_{G i}(0), \beta_{G i}(0), \alpha_{A i}(0)$, and $\beta_{A i}(0)$ by prior distribution, the updating formula of the evaluation criterion function parameters in gyroscope and accelerometer assemblies can be expressed as follows:

$$
\begin{gathered}
H_{G i}(k)=\beta_{G i}(k) \nabla^{2} E_{d G i}\left(w_{G i}^{M P}(k)\right) \\
+\alpha_{G i}(k) \nabla^{2} E_{w G i}\left(w_{G i}^{M P}(k)\right), \\
\gamma_{G i}(k)=M_{G}-2 \alpha_{G i}(k) \operatorname{tr}\left(H_{G i}(k)\right)^{-1}, \\
\alpha_{G i}(k+1)=\frac{\gamma_{G i}(k)}{2 E_{w G i}\left(w_{G i}^{M P}(k)\right)}, \\
\beta_{G i}(k+1)=\frac{N-\gamma_{G i}(k)}{2 E_{d G i}\left(w_{G i}^{M P}(k)\right)}, \\
H_{A i}(k)=\beta_{A i}(k) \nabla^{2} E_{D A i}\left(w_{A i}^{M P}(k)\right) \\
+\alpha_{A i}(k) \nabla^{2} E_{w A i}\left(w_{A i}^{M P}(k)\right), \\
\gamma_{A i}(k)=M_{A}-2 \alpha_{A i}(k) \operatorname{tr}\left(H_{A i}(k)\right)^{-1}, \\
\alpha_{A i}(k+1)=\frac{\gamma_{A i}(k)}{2 E_{w A i}\left(w_{A i}^{M P}(k)\right)}, \\
\beta_{A i}(k+1)=\frac{N-\gamma_{A i}(k)}{2 E_{d A i}\left(w_{A i}^{M P}(k)\right)},
\end{gathered}
$$

where $\gamma_{G i}(k)$ and $\gamma_{A i}(k)$ are called the effective number of parameters. $H_{G i}(k)$ and $H_{A i}(k)$ are the Hessian matrix of the evaluation criterion function.

The training process of Bayesian regularization neural network is iterative algorithm for compensating temperature error of POS. The limited function is

$$
\begin{aligned}
& \left|\alpha_{G i}(k+1)-\alpha_{G i}(k)\right| \leq e_{\alpha G i}, \\
& \left|\beta_{G i}(k+1)-\beta_{G i}(k)\right| \leq e_{\beta G i}, \\
& \left|\alpha_{A i}(k+1)-\alpha_{A i}(k)\right| \leq e_{\alpha A i}, \\
& \left|\beta_{A i}(k+1)-\beta_{A i}(k)\right| \leq e_{\beta A i},
\end{aligned}
$$

where $e_{\alpha G i}, e_{\beta G i}, e_{\alpha A i}$, and $e_{\beta A i}$ are infinitesimals; this is equivalent to the convergence of the evaluation criterion function. When the training process of Bayesian regularization neural network is finished, the optimization of the neural network parameters $w_{j G i}^{*}, \mathbf{C}_{j G i}^{*}, \sigma_{j G i}^{*}$ and $w_{j A i}^{*}, \mathbf{C}_{j A i}^{*}$, $\sigma_{j A i}^{*}$ can be obtained. The final result of temperature errors $\delta \bar{G}_{T i}^{*}$ and $\delta \bar{A}_{T i}^{*}$ can be updated by $w_{j G i}^{*}, \mathbf{C}_{j G i}^{*}, \sigma_{j G i}^{*}$ and $w_{j A i}^{*}$, $\mathrm{C}_{j A i}^{*}, \sigma_{j A i}^{*}$. The temperature error $\delta \bar{G}_{T i}^{*}$ is used to compensate the original output of RLG bias $G_{o i}$ in the $i$ th axes. The temperature error $\delta \bar{A}_{T i}^{*}$ is used to compensate the original 


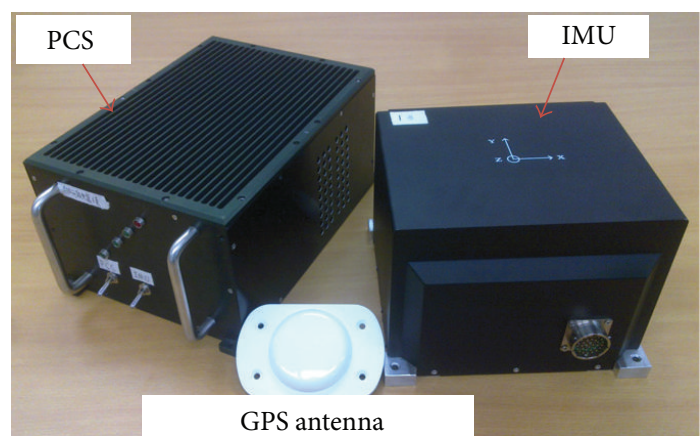

FIgURE 4: The hardware components of developed POS.

output of QMA bias $\bar{A}_{o i}$ in the $i$ th axes. The final output of gyroscope and accelerometer in the $i$ th axes can be given by

$$
\begin{aligned}
G_{b i} & =G_{o i}-\delta \bar{G}_{T i} \\
& =G_{o i}-\left(\sum_{j=1}^{M_{G}} w_{j G i}^{*} \exp \left\{-\frac{\left\|\mathbf{X}_{G i}-\mathbf{C}_{j G i}^{*}\right\|^{2}}{2\left(\sigma_{j G i}^{*}\right)^{2}}\right\}\right), \\
A_{b i} & =A_{o i}-\delta \bar{A}_{T i} \\
& =A_{o i}-\left(\sum_{j=1}^{M_{A}} w_{j A i}^{*} \exp \left\{-\frac{\left\|\mathbf{X}_{A i}-\mathbf{C}_{j A i}^{*}\right\|^{2}}{2\left(\sigma_{j A i}^{*}\right)^{2}}\right\}\right) .
\end{aligned}
$$

Bayesian regularization method reduces the network weight values under the condition of minimizing network training error by new evaluation criterion which eventually improve the generalization ability of neural network.

\section{Temperature Experiment}

4.1. The Equipment in Temperature Experiment. In temperature experiment, we developed the POS (Model TXR20) which is applied in airborne remote sensing system. The POS as shown in Figure 4 can implement gyros biases calibration, initial alignment, inertial navigation algorithms, and so forth. The POS uses an IMU that is sufficient and separated from the PCS and connected to the PCS by a data interface and power cable. The IMU is designed to be small $(210 \mathrm{~mm} \times 200 \mathrm{~mm} \times 142 \mathrm{~mm})$, light $(6.7 \mathrm{Kg})$, and of little power consumption $(20 \mathrm{~W})$. The common output frequency is $100 \mathrm{~Hz}$ (highest being $200 \mathrm{~Hz}$ ). The overall power consumption of POS including IMU and PCS is $28 \mathrm{~W}$, which is commensurate in performance with the POS/AV610. The nominal accuracy of three RLGs ( $X, Y$, and $Z$ gyroscopes) is less than $0.01^{\circ} / \mathrm{h}$ and the nominal accuracy of three QMAs $(X, Y$, and $Z$ accelerometers) is less than $50 \mu \mathrm{g}$.

4.2. The Procedures of the Temperature Experiment. IMU is attached in a temperature chamber as shown in Figure 5. The IMU and temperature chamber are required to be stationary during the whole experiment. The temperature of the chamber varies from $-30^{\circ} \mathrm{C}$ to $50^{\circ} \mathrm{C}$. To establish the temperature error model of POS, an experiment is implemented.

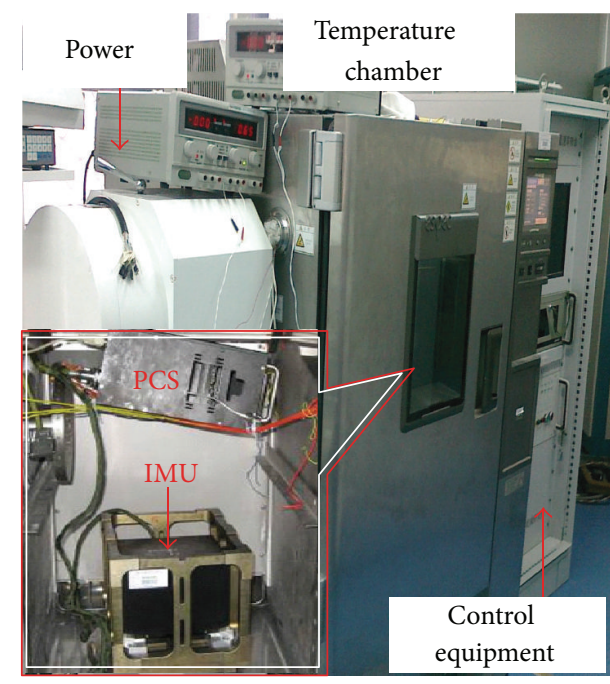

FIGURE 5: The equipment of the temperature experiment.

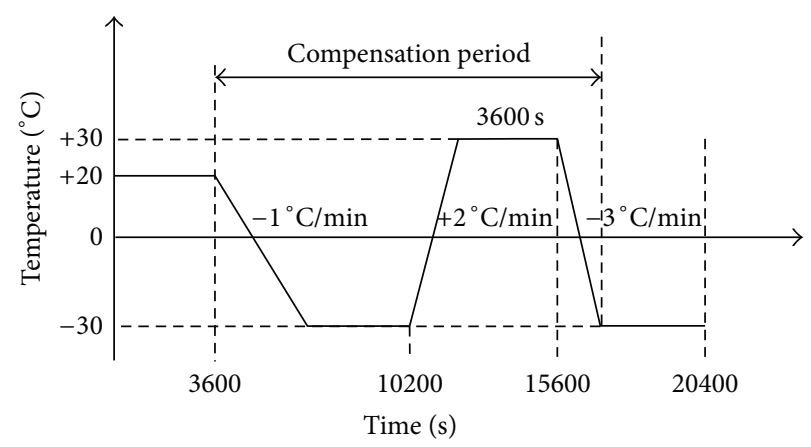

Figure 6: The procedures of the temperature experiment.

Firstly, the temperature is kept at $20^{\circ} \mathrm{C}$ for 1.5 hours to attain thermodynamic equilibrium of RLG and QMA assemblies. Secondly, the temperature of the chamber increases to $50^{\circ} \mathrm{C}$ with uniform rate of $3^{\circ} \mathrm{C} / \mathrm{min}$. Thirdly, the temperature is kept at $50^{\circ} \mathrm{C}$ for 2 hours. Finally, the temperature of the chamber decreases to $-30^{\circ} \mathrm{C}$ with a uniform rate of $-3^{\circ} \mathrm{C} / \mathrm{min}$; then the temperature is also kept for 2 hours.

The temperature errors are chosen as the training target of the RBF neural network, and then it is trained by the traditional RBF neural network and proposed method.

4.3. Compensation Results of Temperature Errors. In order to validate the generalization ability of the RBF neural network trained by the proposed method, a verification test is implemented as shown in Figure 6. Firstly, the temperature is kept at $20^{\circ} \mathrm{C}$ for 1 hour. Secondly, the temperature of the chamber decreases to $-30^{\circ} \mathrm{C}$ with uniform rate of $-1^{\circ} \mathrm{C} / \mathrm{min}$. The temperature is kept at $-30^{\circ} \mathrm{C}$ for 1 hour. Thirdly, the temperature of the chamber increases to $30^{\circ} \mathrm{C}$ with a uniform rate of $2^{\circ} \mathrm{C} / \mathrm{min}$. The temperature is kept at $30^{\circ} \mathrm{C}$ for 1 hour. Finally, the temperature of the chamber decreases to $-30^{\circ} \mathrm{C}$ with a uniform rate of $-3^{\circ} \mathrm{C} / \mathrm{min}$ and is kept at $-30^{\circ} \mathrm{C}$ for 1 hour. 
TABLE 1: The compensational results in gyroscope assembly.

\begin{tabular}{lcccc}
\hline Gyroscope & Original bias $\left({ }^{\circ} / \mathrm{h}\right)$ & Traditional method $\left({ }^{\circ} / \mathrm{h}\right)$ & Proposed method $\left({ }^{\circ} / \mathrm{h}\right)$ & Improvement $(\%)$ \\
\hline$X$ & 0.0298 & 0.0087 & 0.0078 & 10.4 \\
$Y$ & 0.0130 & 0.0104 & 0.0098 & 5.7 \\
$Z$ & 0.0232 & 0.0091 & 0.0080 & 12.1 \\
\hline
\end{tabular}

TABLE 2: The compensational results in accelerometer assembly.

\begin{tabular}{lcccc}
\hline Accelerometer & Original bias $(\mu g)$ & Traditional method $(\mu g)$ & Proposed method $(\mu g)$ & Improvement $(\%)$ \\
\hline$X$ & 181.37 & 24.52 & 18.96 & 22.6 \\
$Y$ & 199.07 & 30.33 & 21.89 & 27.8 \\
$Z$ & 438.58 & 24.13 & 16.58 & 31.3 \\
\hline
\end{tabular}
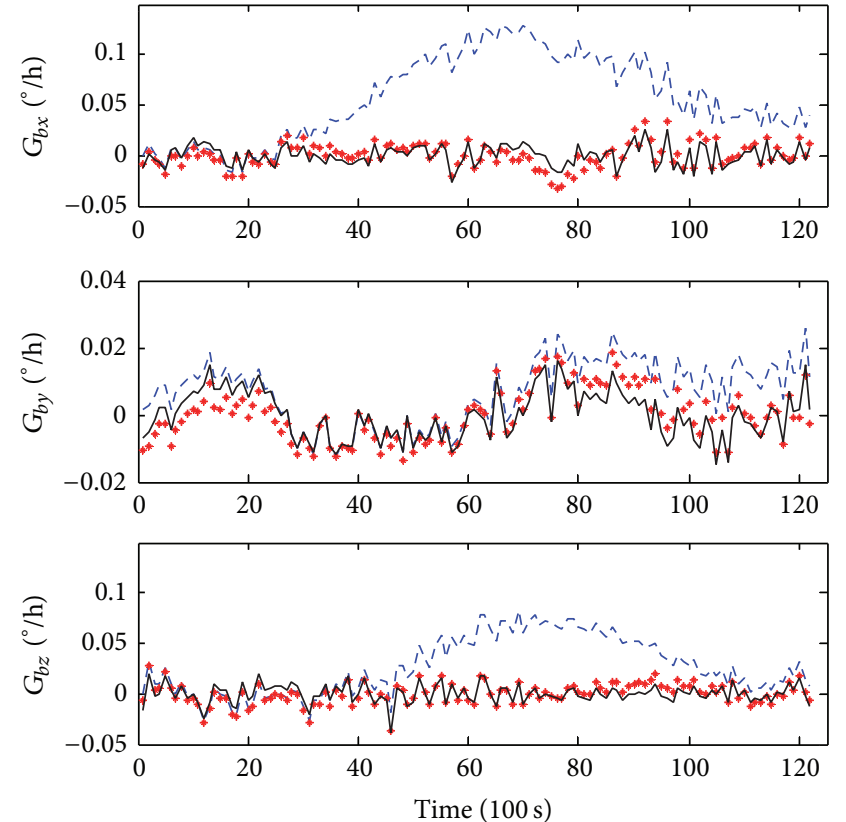

- - Original bias

Traditional method

_ Proposed method

Figure 7: Curves of RLG bias changing with temperature.

The temperature errors of gyroscope and accelerometer assemblies in verification test are compensated by the traditional RBF neural network and proposed method in the paper. The compensation results of gyroscope and accelerometer assemblies are shown in Figures 7 and 8 . The comparisons of results are given in Tables 1 and 2.

As it is evident from Tables 1 and 2, the proposed method reduces the temperature errors of RLG bias and QMA bias effectively.

\section{The Airborne Application Experiment}

In order to further validate the application capability of the proposed method, the airborne application experiment shown in Figure 9 is implemented in July 2013, Weihai City,
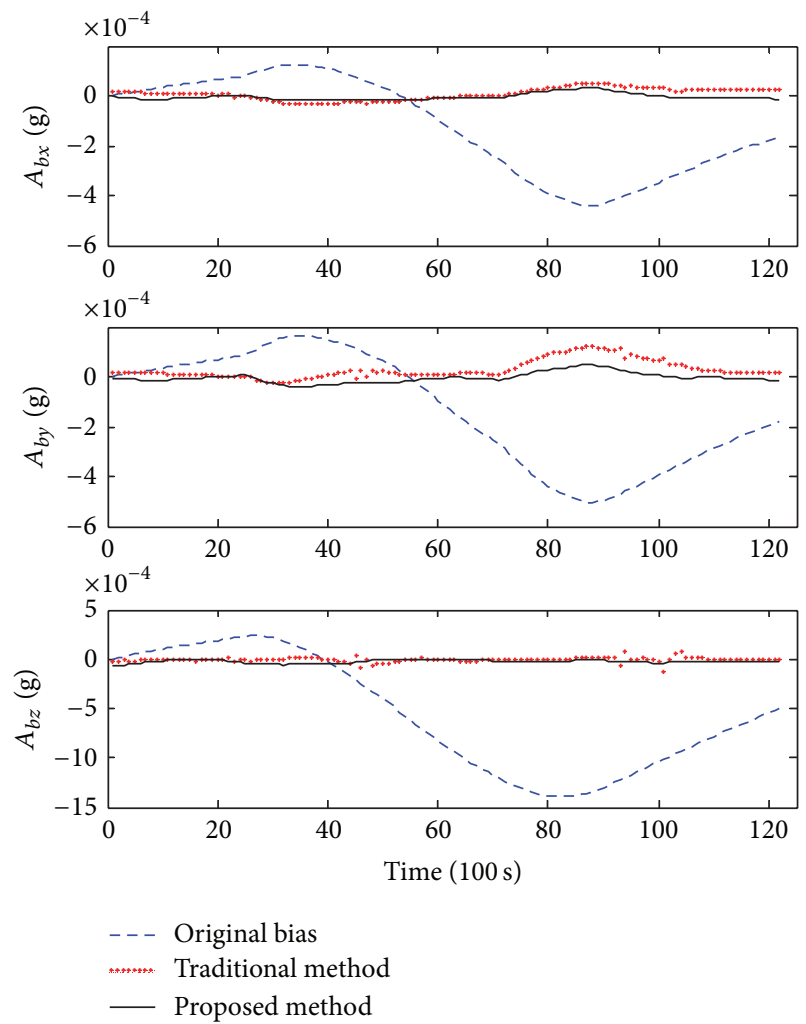

FIGURE 8: Curves of QMA bias changing with temperature.

Shandong, China. The equipment of the airborne application experiments includes an experimental airplane (Y-12), TSMFTIS, and POS. The POS and TSMFTIS are fastened together and then installed on the inertial stabilized platform. The inertial stabilized platform is connected with the cabin of the airplane. Based on the motion information provided by POS, TSMFTIS successfully accomplishes airborne remote sensing assignment.

5.1. The Result of Airborne Experiment. The airplane has a flight of 3 hours and 20 minutes and flies at a height of 2800 meters. The environment temperature changes rapidly throughout the flight. During the 15-minute climbing, the external temperature changes rapidly from $34^{\circ} \mathrm{C}$ to $19^{\circ} \mathrm{C}$, 


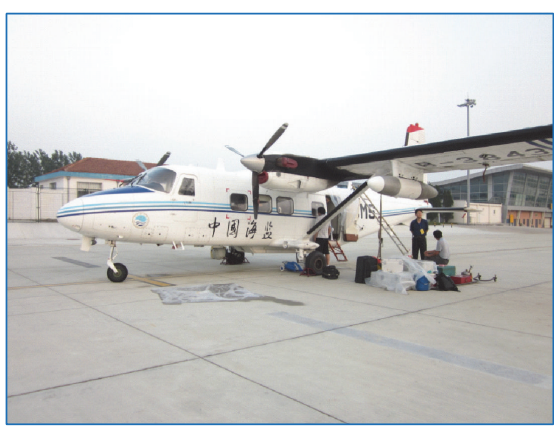

The experimental aircraft applied in remote sensing

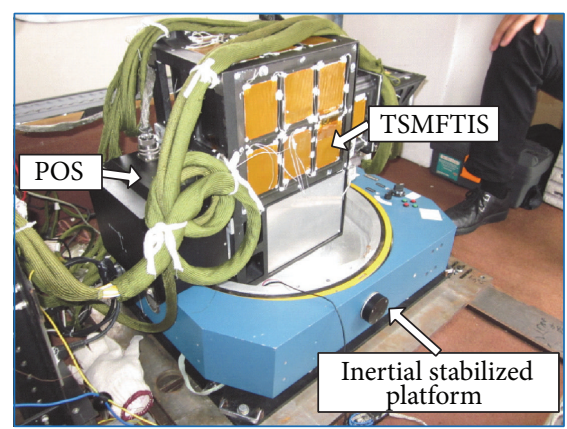

The POS and TSMFTIS in the aircraft

FIGURE 9: The airborne application experiments.

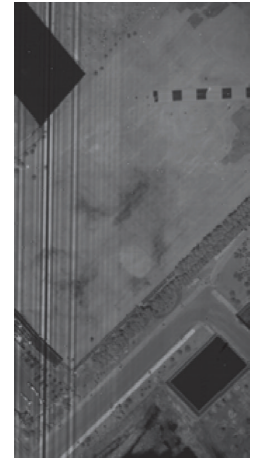

1st frame

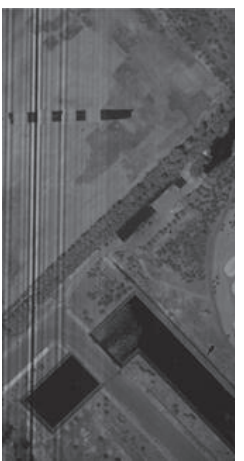

150th frame

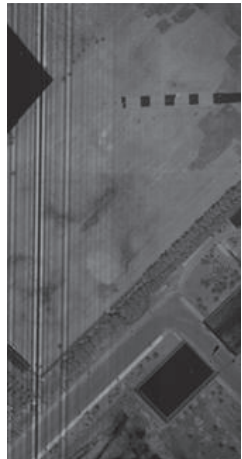

30th frame

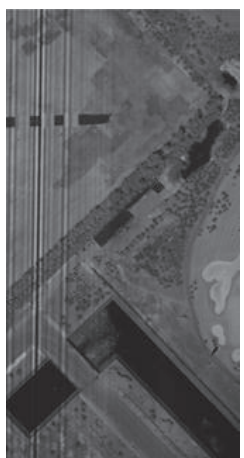

180th frame

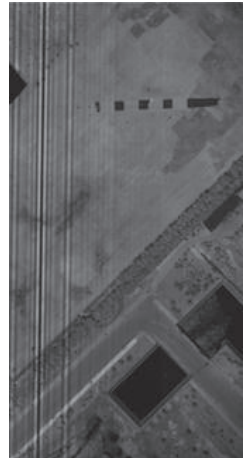

60th frame

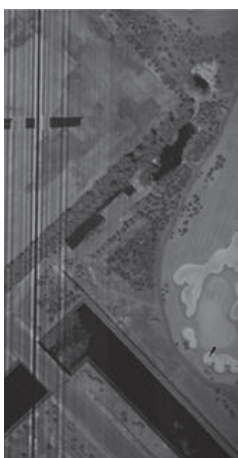

210th frame

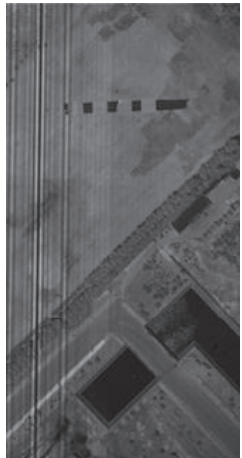

120th frame

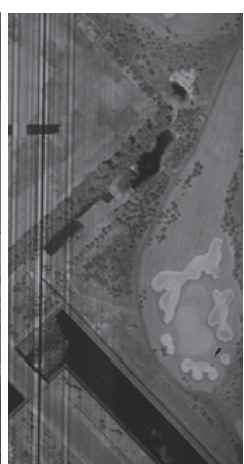

240th frame

Figure 10: The interferogram with TSMFTIS using POS data.

which affects the measurement accuracy of inertial navigation. Accordingly, temperature error compensation is necessary in inertial navigation. The inertial navigation error results by traditional RBF neural network method and the proposed method are shown in Table 3.

The results show that compared to the traditional RBF neural network method, the inertial navigation error of proposed method is reduced to 0.57 nautical mile from 0.63 nautical mile during 1 hour, falling by $9.52 \%$. The inertial navigation error over 3 hours and 20 minutes is reduced to 1.60 nautical miles from 1.73 nautical miles, falling by $7.51 \%$.
5.2. The Airborne Remote Sensing Experiment of TSMFTIS with POS. The application of TSMFTIS, which plays a vital role in the remote sensing system, has no internal moving parts and high throughput advantages [19]. It is usually applied in satellite remote sensing assignment which requires high stability of the platform to provide accurate position, velocity, and attitude references for TSMFTIS to measure the geographic position and orientation directly during the push-broom process [20]. However, in airborne remote sensing system, the attitude changes of the aircraft due to air disturbance cannot be completely compensated 


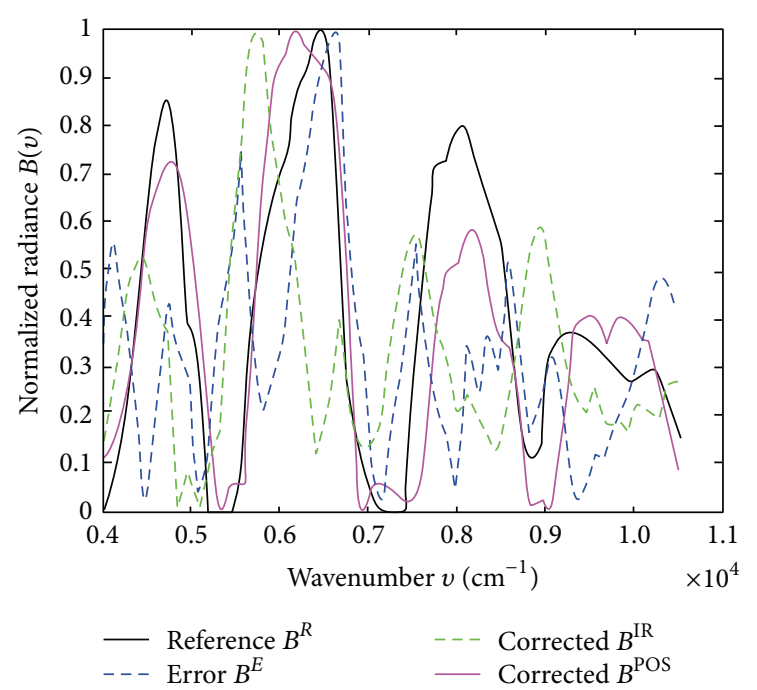

(a)
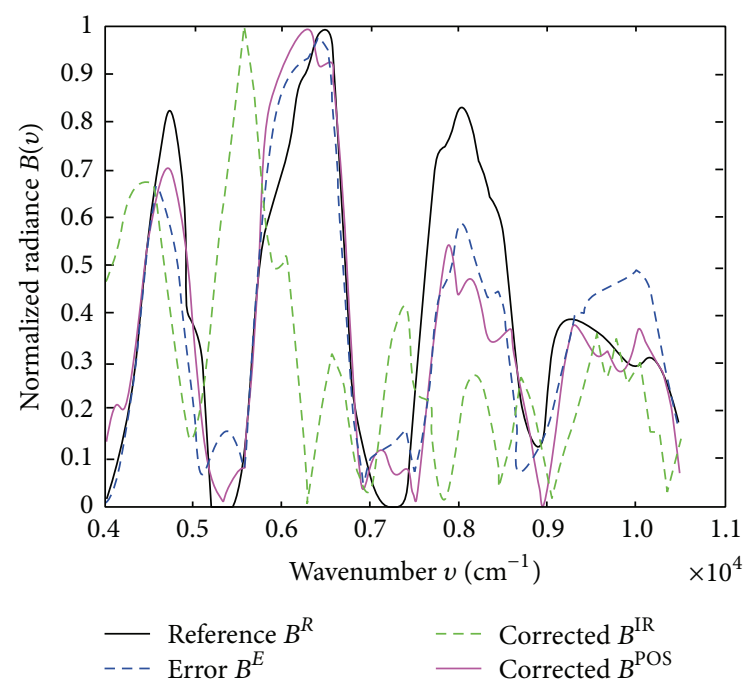

(c)

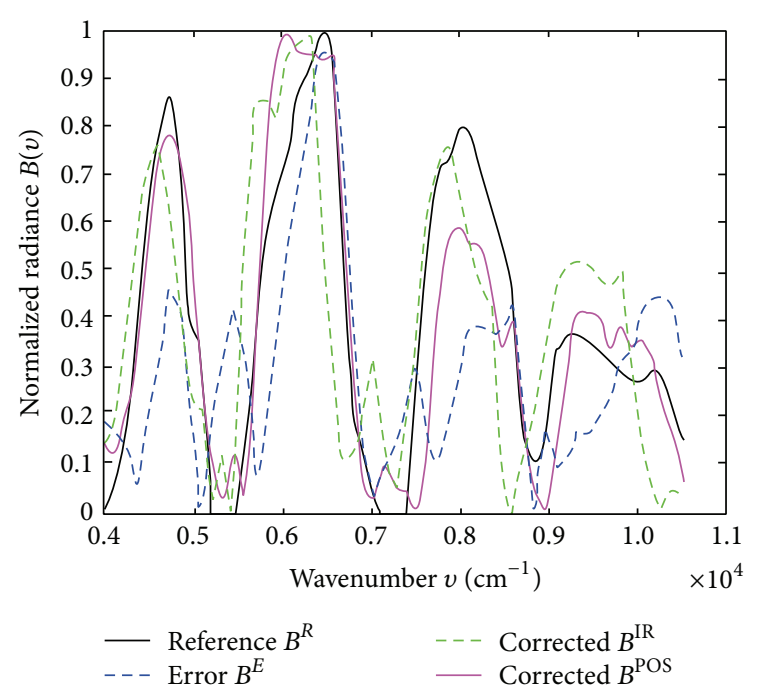

(b)

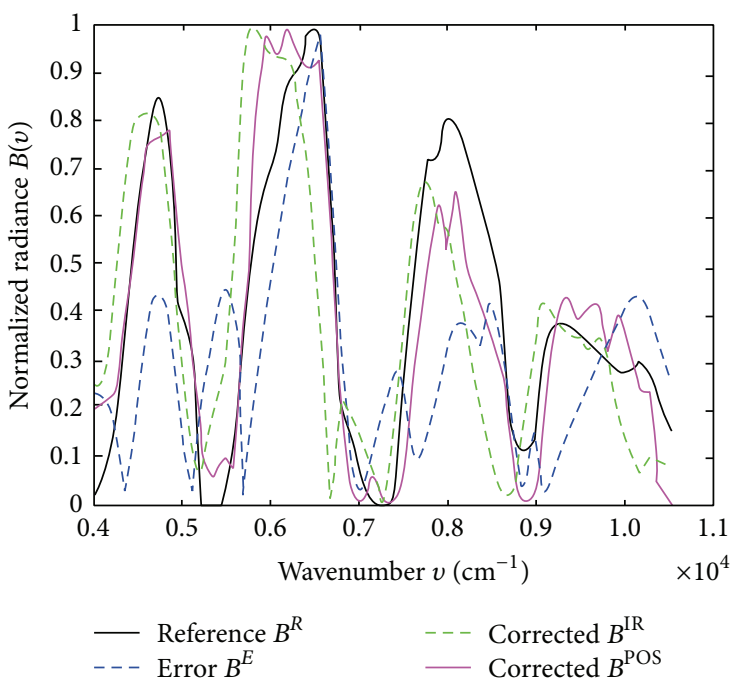

(d)

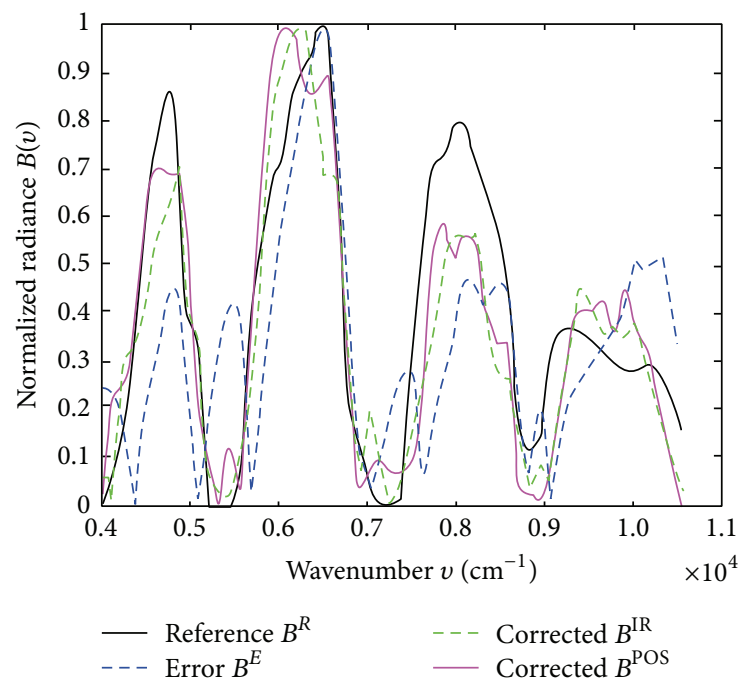

(e)

FIGURE 11: Five groups of the reconstructed spectra. 
TABLE 3: Inertial navigation error in the airborne experiments.

\begin{tabular}{lccc}
\hline & Traditional RBFNN & Proposed method & Improvement in precision \\
\hline $\begin{array}{l}\mathbf{1} \text { hour of navigation error } \\
\text { (nautical mile) }\end{array}$ & 0.63 & 0.57 & $9.52 \%$ \\
$\begin{array}{l}3 \text { hours 20 minutes of } \\
\text { navigation error (nautical mile) }\end{array}$ & 1.73 & 1.60 & $7.51 \%$ \\
\hline
\end{tabular}

TABLE 4: The RQE results in airborne remote sensing experiment.

\begin{tabular}{lcccc}
\hline Group & Traditional method & Image registration & Target-tracking-based & Improvement (\%) \\
\hline 1 & 0.6410 & 0.7275 & 0.3141 & 51.00 \\
2 & 0.4930 & 0.4407 & 0.2776 & 43.69 \\
3 & 0.6450 & 0.3474 & 0.2608 & 59.56 \\
4 & 0.4749 & 0.4799 & 0.2637 & 44.47 \\
5 & 0.4697 & 0.2893 & 0.2761 & 41.22 \\
\hline Average & 0.5447 & 0.4569 & 0.2784 & 47.99 \\
\hline
\end{tabular}

by the inertial stabilized platform because of its limit on stability and precision which cannot completely compensate the deviations. Therefore, the aircraft cannot be regarded as a stable platform compared with satellites platforms, which restricts the application of TSMFTIS in airborne remote sensing; sometimes even the interferogram cannot be acquired. This experiment, as an innovative attempt of the application of TSMFTIS on airborne remote sensing system, efficiently achieves target-tracking-based method, which considers POS data as its vital element [21].

POS data provides attitude information on TSMFTIS in remote sensing imaging, which as a result tracks the position of the target on each recorded image and obtains the right target interferogram for airborne TSMFTIS. The right target interferogram by target-tracking-based method using POS data, which is obtained from the proposed method, is shown in Figure 10. However, the other methods cannot obtain all the effective interferograms without POS data.

In Figure 11, the reconstructed spectrum from the reference interferograms by spectroradiometer is denoted by reference spectrum $B^{R}$, while $B^{E}$ is the error spectrum reconstructed from distorted interferogram. $B^{\mathrm{IR}}$ and $B^{\mathrm{POS}}$ represent the spectra reconstructed from the corrected interferogram based on image registration method and targettracking-based method obtained by POS data, respectively $[22,23]$. The reconstructed spectrum using POS data matches the reference spectrum better than that from image registration method.

The relative spectral quadratic error (RQE) [24] result shown in Table 4 is regarded as an evaluation standard of spectral precision. Table 4 demonstrates that the accuracy of the reconstructed spectrum by target-tracking-based method using POS data improves rapidly compared with traditional method in all five experiments, which validates both the effectiveness and robustness of the proposed method. The average result shows that the RQE is reduced to 0.2784 from 0.5447 , falling by $47.99 \%$.

\section{Conclusion}

POS plays a vital role and becomes a research and development focus in airborne remote sensing systems. In this paper, a nonlinear multiparameters temperature error model and compensation method of POS based on Bayesian regularization neural network were proposed to improve the measurement precision of POS. At first, the nonlinear multiparameters temperature error model of gyroscope and accelerometer assemblies of POS was established. Then, a Bayesian regularization neural network method was proposed to improve the generalization ability of the network, which could be used as the evaluation criterion to optimize the configuration of temperature error. The experiment results show that the compensated temperature errors of gyroscope and accelerometer assemblies are reduced compared to the traditional method. The proposed method had been applied in postprocessing algorithm of POS, which successfully accomplished the first airborne TSMFTIS remote sensing assignment.

\section{Conflict of Interests}

The authors declare that there is no conflict of interests regarding the publication of this paper.

\section{Acknowledgments}

This work was supported in part by the National Program on Key Basic Research Projects of China under Grant 2009CB724002, the CAST Innovation Foundation CAST201205, the Beijing Youth Elite Project, the Basic Scientific Research YWF-14-YQGD-001, the National Special Instrument Project 2012YQ160185, the Science and Technology Project of China Southern Grid 20100002012117034, and in part by the National Natural Science Foundation of China under Grants 60825305, 61121003, and 61104198. 


\section{References}

[1] J. C. Fang and S. Yang, "Study on innovation adaptive EKF for in-flight alignment of airborne POS," IEEE Transactions on Instrumentation and Measurement, vol. 60, no. 4, pp. 1378-1388, 2011.

[2] M. M. R. Mostafa and K.-P. Schwarz, "Digital image georeferencing from a multiple camera system by GPS/INS," ISPRS Journal of Photogrammetry and Remote Sensing, vol. 56, no. 1, pp. 1-12, 2001.

[3] J. Li, J. Fang, and M. Du, "Error analysis and gyro-bias calibration of analytic coarse alignment for airborne POS," IEEE Transactions on Instrumentation and Measurement, vol. 61, no. 11, pp. 3058-3064, 2012.

[4] H.-S. Ahn and C.-H. Won, "DGPS/IMU integration-based geolocation system: Airborne experimental test results," Aerospace Science and Technology, vol. 13, no. 6, pp. 316-324, 2009.

[5] J. Cheng and J. Fang, "Comparison of compensation methods on RLG temperature error and their application in POS," in Proceedings of the 8th IEEE International Symposium on Instrumentation and Control Technology (ISICT '12), pp. 189194, July 2012.

[6] C. Guo, Y. Xu, and X. Zhao, "Investigation on the temperature compensating model ring laser gyroscope," Chinese Optics Letters, vol. 4, no. 10, pp. 576-579, 2006.

[7] G. Wei, G. Li, Y. Wu, and X. Long, "Application of Least Squares-Support Vector Machine in system-level temperature compensation of ring laser gyroscope," Measurement, vol. 44, no. 10, pp. 1898-1903, 2011.

[8] Q. Zhang, X.-F. Liu, J. Zhan, and G.-M. Chen, “Temperature modeling study for high precision gyroscope based on neural network," in Proceedings of the International Symposium on Intelligent Ubiquitous Computing and Education (IUCE '09), pp. 85-87, May 2009.

[9] R. Sharaf and A. Noureldin, "A neural network model of optical gyros drift errors with application to vehicular navigation," in Applications of Digital Image Processing XXVII, vol. 5558 of Proceedings of SPIE, pp. 13-20, August 2004.

[10] Q. Hua, Y. Gao, X.-Z. Wang, and B.-Y. Zhao, "A new approach to improving generalization ability of feed-foward neural networks," in Proceedings of the International Conference on Machine Learning and Cybernetics (ICMLC '10), pp. 1413-1419, July 2010.

[11] J. Li, A. Chen, J. Fang, and J. Cheng, "Time delay modeling and compensation of dithered RLG POS with antivibrators and filter," Measurement, vol. 46, no. 6, pp. 1928-1937, 2013.

[12] J. Li, J. Fang, and S. S. Ge, "Kinetics and design of a mechanically dithered ring laser gyroscope position and orientation system," IEEE Transactions on Instrumentation and Measurement, vol. 62, no. 1, pp. 210-220, 2013.

[13] E. Lithopoulos, B. Reid, and B. Scherzinger, "The position and orientation system (POS) for survey applications," in Proceedings of the International Archives of Photogrammetry and Remote Sensing, pp. 467-471, 1996.

[14] J. S. Cain, D. A. Staley, G. R. Heppler, and J. McPhee, "Stability analysis of a dynamically timed gyroscope," Journal of Guidance, Control, and Dynamics, vol. 29, no. 4, pp. 965-969, 2006.

[15] L. Franco, J. M. Jerez, and J. M. Bravo, "Role of function complexity and network size in the generalization ability of feedforward networks," in Proceedings of the 8th International
Workshop on Artificial Neural Networks (IWANN '05), pp. 1-8, June 2005.

[16] J. L. Ticknor, "A Bayesian regularized artificial neural network for stock market forecasting," Expert Systems with Applications, vol. 40, no. 14, pp. 5501-5506, 2013.

[17] E. Rank, "Application of Bayesian trained RBF networks to nonlinear time-series modeling," Signal Processing, vol. 83, no. 7, pp. 1393-1410, 2003.

[18] P. Kumar, S. N. Merchant, and U. B. Desai, "Improving performance in pulse radar detection using Bayesian regularization for neural network training," Digital Signal Processing, vol. 14, no. 5, pp. 438-448, 2004.

[19] L. J. Su, Y. Yuan et al., "Spectrum reconstruction method for airborne temporally-spatially modulated Fourier transform imaging spectrometers," IEEE Transactions on Geoscience and Remote Sensing, vol. 52, no. 6, pp. 3720-3728, 2014.

[20] J. Reguera-Salgado, M. Calvino-Cancela, and J. Martin-Herrero, "GPU geocorrection for airborne pushbroom imagers," IEEE Transactions on Geoscience and Remote Sensing, vol. 50, no. 11, pp. 4409-4419, 2012.

[21] Y. Yuan, X. Zhang, C. Sun, and Z. Zhou, "Modeling of the temporally and spatially modulated fourier transform imaging spectrometer working in orbit," Optik, vol. 122, no. 17, pp. 15761583, 2011.

[22] X. Fan, H. Rhody, and E. Saber, "A spatial-feature-enhanced MMI algorithm for multimodal airborne image registration," IEEE Transactions on Geoscience and Remote Sensing, vol. 48, no. 6, pp. 2580-2589, 2010.

[23] P. Vandewalle, S. Süsstrunk, and M. Vetterll, "A frequency domain approach to registration of aliased images with application to super-resolution," EURASIP Journal on Applied Signal Processing, vol. 2006, Article ID 71459, 14 pages, 2006.

[24] C. Mailhes, P. Vermande, and F. Castanié, "Spectral image compression," Journal of Optics, vol. 21, no. 3, pp. 121-132, 1990. 


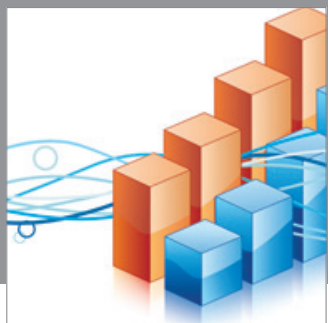

Advances in

Operations Research

mansans

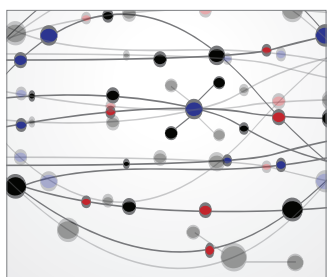

The Scientific World Journal
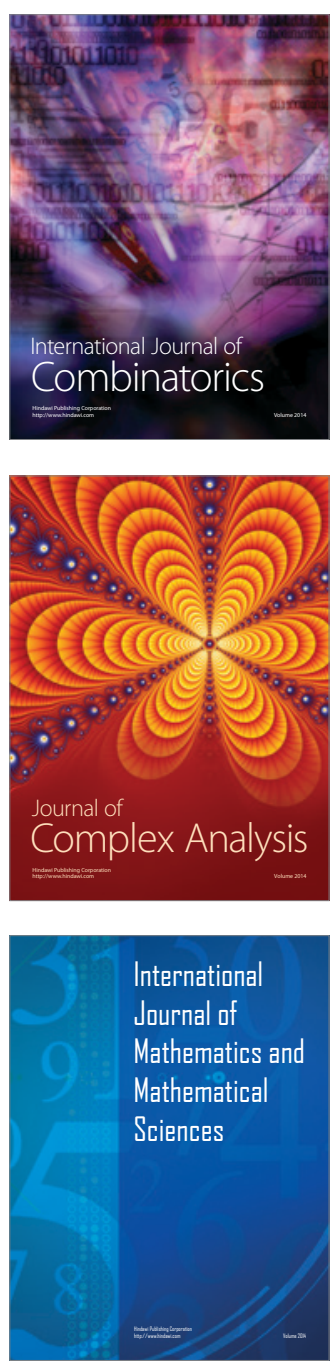
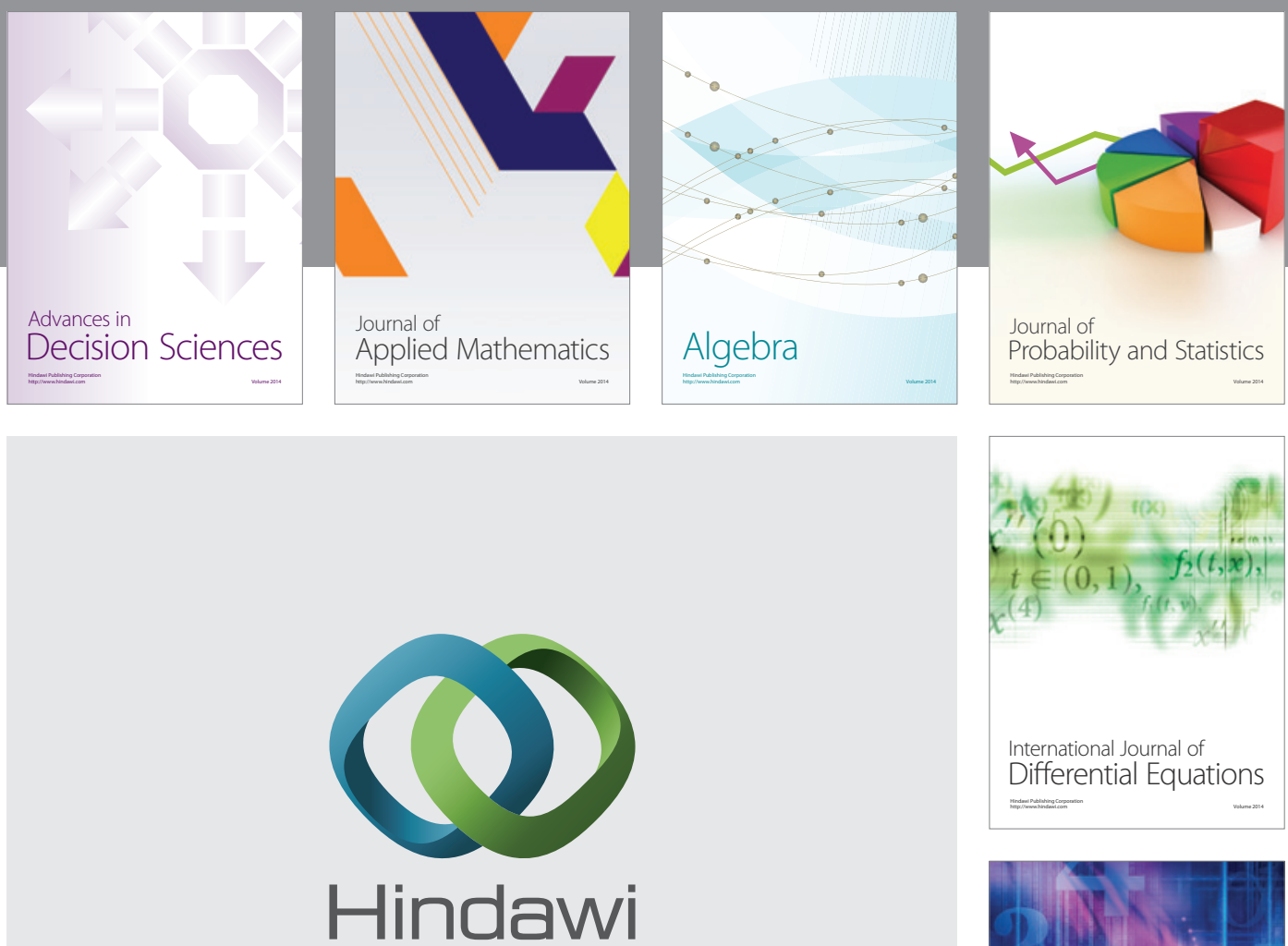

Submit your manuscripts at http://www.hindawi.com
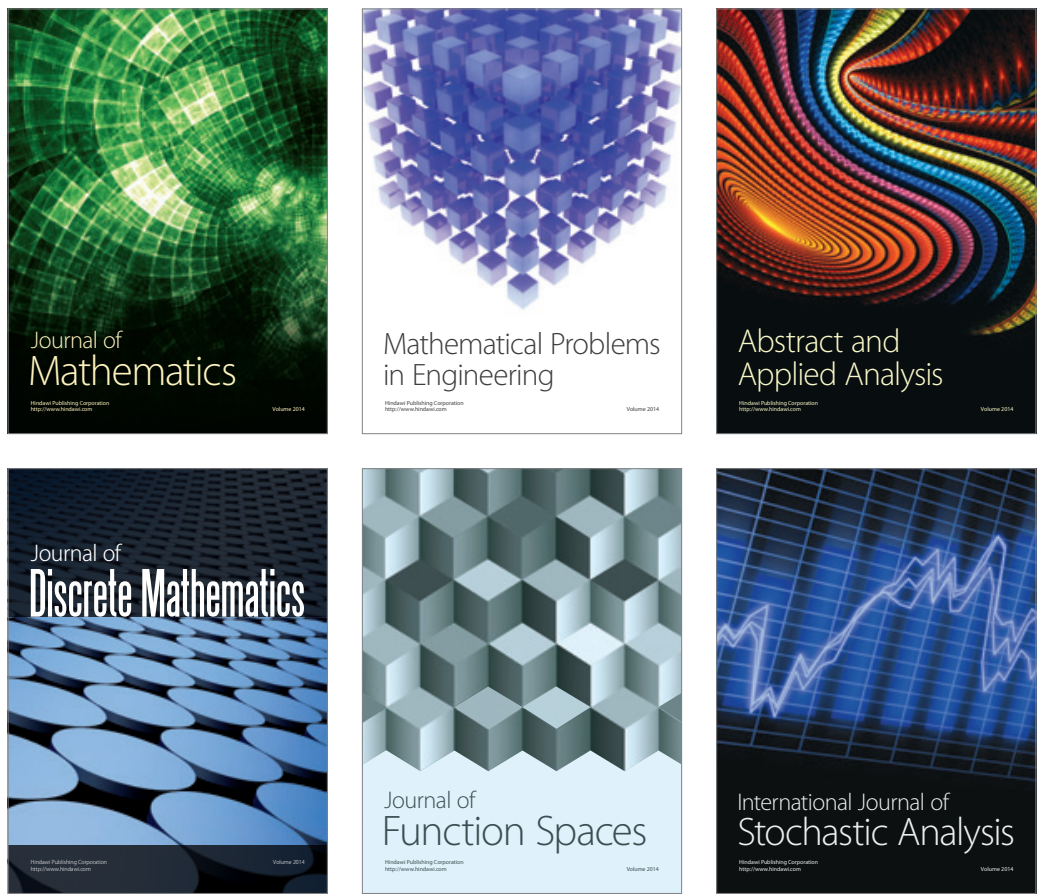

Journal of

Function Spaces

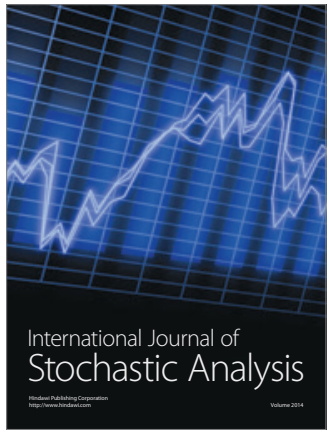

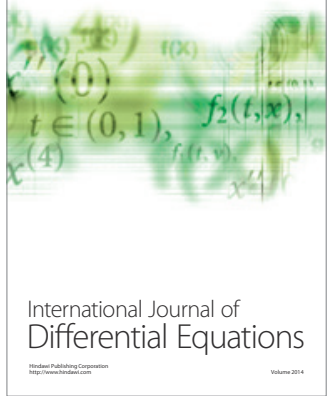
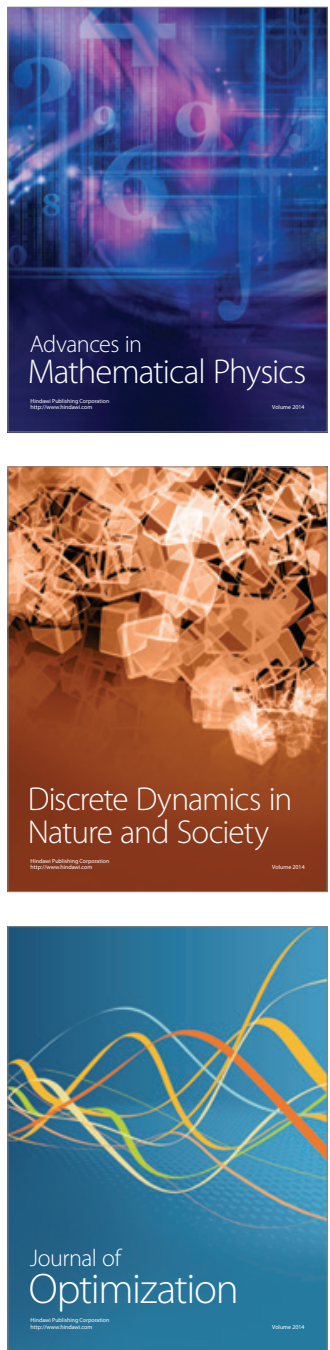